
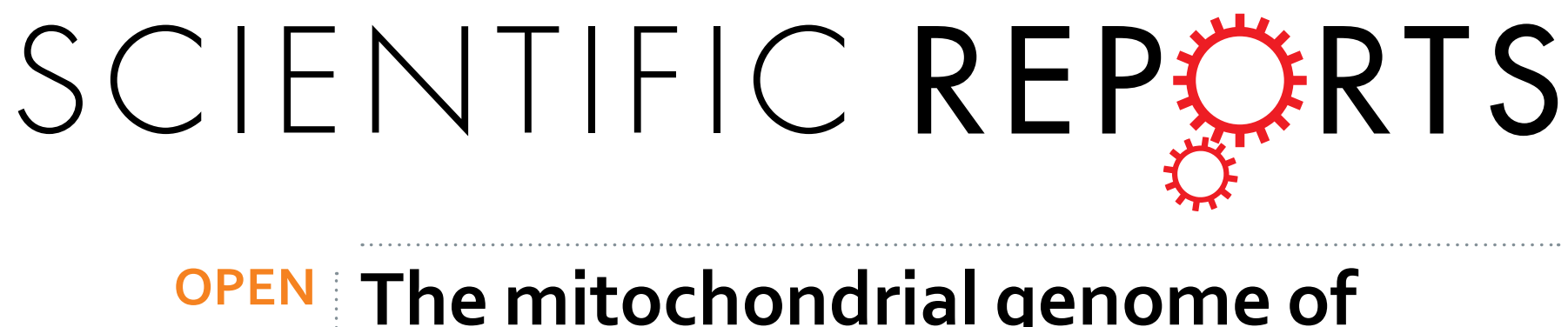

The mitochondrial genome of booklouse, Liposcelis sculptilis (Psocoptera: Liposcelididae) and

Received: 29 April 2016

Accepted: 07 July 2016

Published: 29 July 2016

\section{the evolutionselis}

\author{
Yan Shi, Qing Chu, Dan-Dan Wei, Yuan-Jian Qiu, Feng Shang, Wei Dou \& Jin-Jun Wang
}

Bilateral animals are featured by an extremely compact mitochondrial (mt) genome with 37 genes on a single circular chromosome. To date, the complete mt genome has only been determined for four species of Liposcelis, a genus with economic importance, including L. entomophila, L. decolor, $L$. bostrychophila, and $L$. paeta. They belong to A, B, or D group of Liposcelis, respectively. Unlike most bilateral animals, L. bostrychophila, L. entomophila and $L$. paeta have a bitipartite mt genome with genes on two chromosomes. However, the mt genome of $L$. decolor has the typical $m t$ chromosome of bilateral animals. Here, we sequenced the mt genome of $L$. sculptilis, and identified 35 genes, which were on a single chromosome. The mt genome fragmentation is not shared by the $D$ group of Liposcelis and the single chromosome of $L$. sculptilis differed from those of booklice known in gene content and gene arrangement. We inferred that different evolutionary patterns and rate existed in Liposcelis. Further, we reconstructed the evolutionary history of 21 psocodean taxa with phylogenetic analyses, which suggested that Liposcelididae and Phthiraptera have evolved $134 \mathrm{Ma}$ and the sucking lice diversified in the Late Cretaceous.

During the last two decades, the booklice of the genus Liposcelis have emerged as serious pests of stored commodities worldwide ${ }^{1}$. Moreover, many microorganisms, such as rickettsial species have been found inside and on outside surfaces of booklice bodies, and these could be transferred to humans thereby posing a threat to human health ${ }^{2,3}$.

Recently, several types of atypical mitochondrial ( $\mathrm{mt}$ ) genome organization have been reported in psocodean insects (superorder Psocodea). Psocodea contains two orders of insects: Psocoptera (booklice and barklice) and Phthiraptera (chewing and sucking lice). The mt genomes of human lice, Pediculus humanus, P. capitis and Pthirus pubis, consist of 14 to 20 mini-chromosomes, each is 1.8 to $4 \mathrm{~kb}$ in size and contains one to five genes ${ }^{4,5}$, while nad4 was unidentified in Pthirus pubis ${ }^{5}$. Haematomyzus elephantis from the suborder Rhynchophthirina was sequenced with ten minichromosomes ${ }^{6}$, and nad 2 and three tRNA were unidentified. The chewing louse, Coloceras sp., has a typical mitochondrial chromosome with 37 genes and a circular mt DNA molecule that is approximately half the size of the typical $\mathrm{mt}$ chromosome $\mathrm{e}^{7}$. The booklice, L. bostrychophila, has a bipartite $\mathrm{mt}$ genome with two chromosomes: one chromosome is $<8 \mathrm{~kb}$ in size and has 16 genes and the other is $<8.5 \mathrm{~kb}$ in size and has 22 genes $^{8}$. The $\mathrm{mt}$ genome of $L$. decolor has the typical mitochondrial chromosome of bilateral animals, 14,405 bp long with 37 genes (13 PCGs, 22 tRNAs and 2 rRNAs). For L. entomophila and L. paeta, one mitochondrial chromosome has most of the mt genes whereas the other chromosome has largely pseudogenes and non-coding regions ${ }^{1}$. Intriguingly, nad $4 L$ was absent in L. entomophila ${ }^{1}$. L. entomophila and L. paeta differ substantially from each other and from L. bostrychophila in gene content and gene arrangement in their mt chromosomes, although they have evolved multipartite mt genomes. However, a recent influential study has discovered maternally transmitted sex ratio distortion in L. nr. bostrychophila that is associated with extraordinarily

Key Laboratory of Entomology and Pest Control Engineering, College of Plant Protection, Southwest University, Chongqing 400716, China. Correspondence and requests for materials should be addressed to J.-J.W. (email: wangjinjun@swu.edu.cn) 
divergent mitochondria. Sequencing the $\mathrm{mt}$ genomes of distorter and normal individuals proved to be quite a surprise. Not only were they incredibly divergent, they also had radically different gene order and genome structure. Both distorter and normal individuals had multipartite mt genomes, consisting of at least five and seven minicircles, respectively ${ }^{9}$. Moreover, $L$. decolor, the arrangement of genes differs substantially from that observed in Liposcelis species and other insects ${ }^{10}$. The genus Liposcelis was classified into four groups (A, B, C and D) and has 126 known species worldwide ${ }^{11}$. The booklouse, L. sculptilis, investigated in the current study, belongs to D group. In present, L. bostrychophila and L. paeta of D group, bipartite $\mathrm{mt}$ genome with two chromosomes, have been sequenced. Do all the species from D group have the two mitochondrial chromosomes structure? Based on the hypothesis, we sequenced the mt genome of L. sculptilis.

Despite being classified as Psocoptera, the suborder Psocomorpha is phylogenetically more closely related to the Phthiraptera than to the another suborder Trogiomorpha ${ }^{1,10,12}$. Because the fossil record for lice is poor ${ }^{13}$, there was only few studies that attempted to the evolutionary history among Psocodea suborders and among Anoplura families ${ }^{14,15}$. In addition, they only used the portion of the $\operatorname{cox} 1,18 S$ and $E F-1$ gene sequences to reconstruct the phylogenetic relationship and evolutionary history ${ }^{14,15}$. This study is the first attempt to use mt genome to elucidate the evolutionary history of this unique group.

\section{Results}

Mitochondrial genome of Liposcelis sculptilis. The mt genome of L. sculptilis has one typical circular chromosome (Fig. 1), unlike the other two species from D group of booklice, including L. bostrychophila and L. paeta. The size and the circular organization of the mt chromosome of L. sculptilis was confirmed by two overlapping PCR amplicons, $9.1 \mathrm{~kb}$ (S9-S10 from $c o b$ to $r r n L$ ) and $5.5 \mathrm{~kb}$ in size (S11-S12 from $\mathrm{rrnL}$ to $c o b$ ), respectively (Fig. 1, S1; Table 1). The two amplicons overlapped by $42 \mathrm{bp}$ in cob and $74 \mathrm{bp}$ in rrnL. We found 35 of the 37 genes typically for bilateral animals in L. sculptilis mt genome, and it contains 12 protein-coding genes (atp6, atp8, cob, cox1-cox3, nad1-nad6), two rRNA genes ( $r r n L, r r n S)$ and 21 tRNA genes.

The variation structure of tRNA in L. sculptili. Most tRNAs of the L. sculptilis have the typical clover leaf shaped secondary structure. However, five tRNAs have atypical structures. For T $\Psi \mathrm{C}$ arm and DHU arm, tRNA-His, tRNA-Glu, tRNA-Met, and tRNA-Pro lack the T $\Psi C$ arm, and tRNA-Ser1 lack DHU arm in the L. sculptilis (Fig. 2). In most insects, tRNA-Ser1 also lacks the DHU arm. In terms of anti-codon, 16 tRNAs use common antisense codons, however, five tRNAs (tRNA-Asn, tRNA-His, tRNA-Ile, tRNA-Lys, tRNA-Ser1) use uncommon antisense codons. UUU, AUG, GAU, UUU, and UCU replaced the traditional codons GUU, GUG, AAU, CUU, and GCU, respectively. Secondary structure of 19 tRNAs, 62 mismatches, have base mismatch. There are 38 G-U mismatches, five U-C mismatches, six G-A mismatches, four U-U mismatches, four A-A mismatches, and four A-C mismatches.

Mitochondrial gene codon usage of the booklice. To date, three mt genomes of L. bostrychophila, L. sculptilis and L. paeta from D group of Liposcelis have been sequenced. The A + T content of the L. sculptilis $\mathrm{mt}$ genome was $76.5 \%$, which was higher than L. paeta $(75.23 \%)$ and L. bostrychophila (68.63\%), respectively. The higher A + T content of L. sculptilis was present in all regions, both genes and noncoding regions. Phe (F), Ile (I) and Leu (L) are most frequently used and the frequency used of the two codons of Leu are obviously different in the five Liposcelis species (Fig. 3). In these booklice, the frequency of L2 (UUA and UUG) was significantly higher than that of L1(CUN). However, this result was just the opposite in L. bostrychophila (Fig. 3). Moreover, the difference of $\mathrm{A}+\mathrm{T}$ content between the five booklice were also reflected further in the codon usage: the relative synonymous codon usages (RSCU) of the five booklice showed that L. sculptilis used more NNA and NNT codon than L. paeta and L. bostrychophila. The nucleotide composition of mt genome is usually conserved within a group; however, it varied among L. sculptili, L. paeta and L. bostrychophila. This variation might be related to mt genome fragmentation, because all of the psocodean fragmented mt genomes have a lower $\mathrm{A}+\mathrm{T}$ content ${ }^{10}$.

In total of $63 \mathrm{mt}$ protein-coding genes in five Liposcelis species, the traditional codon ATN (60) were used as start codon. However, the cox 1 of L. decolor and the cox 3 of L. paeta use TTG and the atp 8 of L. bostrychophila use GTG as start codon. In terms of the stop codon, the codon TAA and TAG were used as stop codon in 61 genes. However, incomplete stop codon was used for the nad 1 of L. bostrychophila and the nad $4 L$ of $L$. paeta, respectively (Table 2).

Mitochondrial gene order of the Liposcelis. In the present work, the five Liposcelis species belong to three groups (A, B, and D). L. bostrychophila, L. paeta and L. sculptilis belong to D group, and their phylogenetic relationship has been demonstrated in our phylogenetic trees. However, they have huge differences in the size, gene content, gene order and architecture of the mt genome. The mt gene arrangement in Liposcelis differs substantially from that of the hypothetical ancestor of insects and from that of the barklice (Fig. 4). With the exception of L. entomophila, there is only atp8-atp6 gene block shared between booklice and barklice, even though these five booklice belong to the same genus. In D group of Liposcelis, we found that L. bostrychophila, L. paeta and L. sculptilis shared two gene blocks: atp8-atp6, $\operatorname{cox} 3$-cox1 (Fig. 4).

Phylogenetic relationships and divergence times of the Psocodea. We tested the phylogenetic relationships among the major lineages of the Psocodea together with the mt genome sequences of L. sculptilis, and twenty other psocodean species (Fig. 5). Based on the two different datasets, we recovered two major clades in the Psocodea with strong support values regardless the dataset and the method we used: 1) species of barklice in the suborders Psocomorpha were clustered together; 2) the booklice formed a clade with the parasitic lice (Fig. 5). The parasitic lice (Phthiraptera) are monophyletic with strong support; however, within the parasitic lice, the suborder Ischnocera is paraphyletic and the sister-group relationship between Anoplura and Rhyncophthirina was also strongly supported. For the divergence times of the Psocodea, our results showed that Liposcelididae 


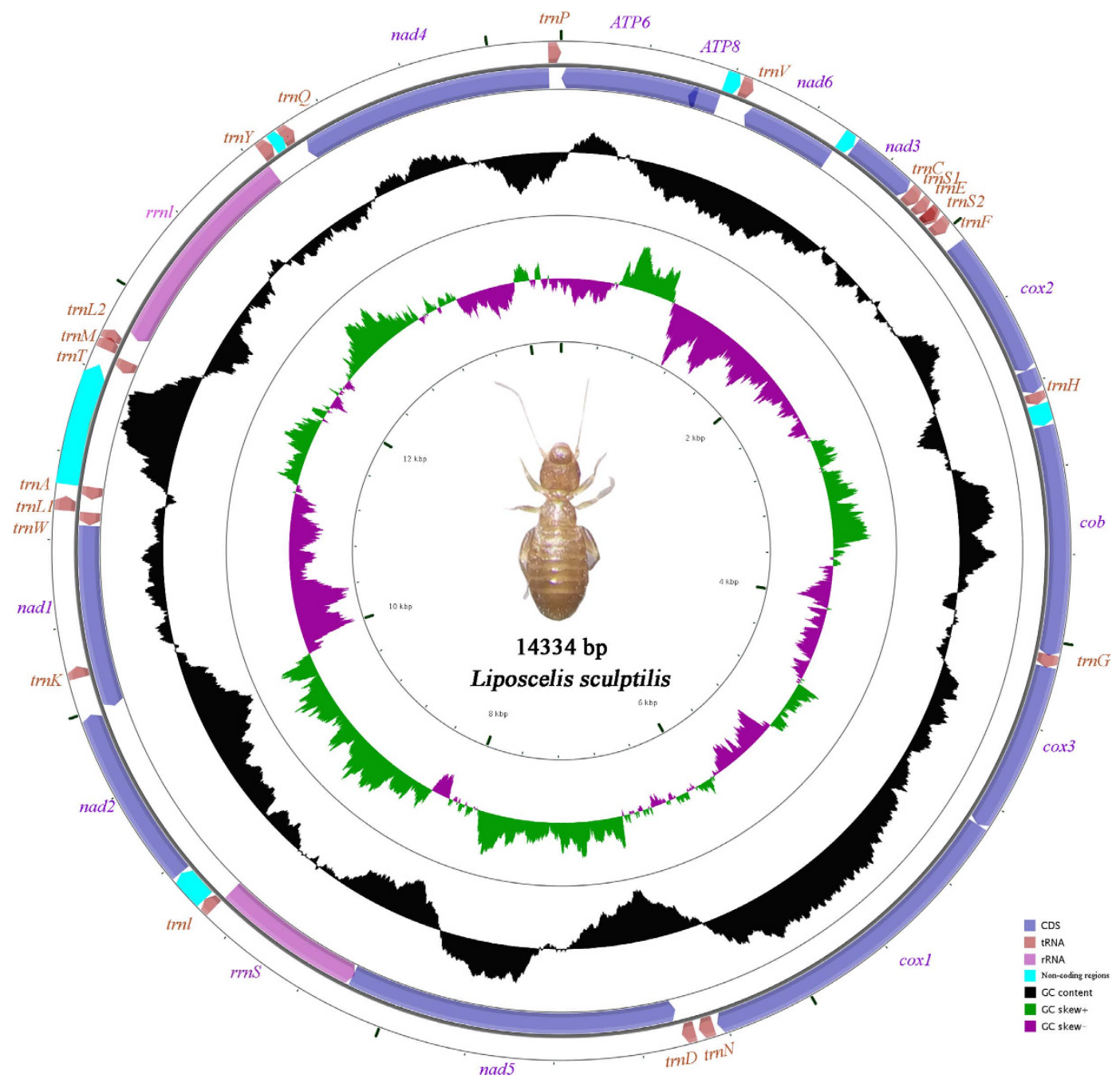

Figure 1. Mitochondrial genomes of Liposcelis sculptilis. Circular maps were drawn with CGView ${ }^{55}$. Arrows indicate the orientation of gene transcription. Protein-coding genes are shown as blue arrows, rRNA genes as purple arrows, tRNA genes as brown arrows and non-coding regions as light green. Abbreviations of gene names are: atp 6 and atp 8 for ATP synthase subunits 6 and 8, cox1-3 for cytochrome oxidase subunits 1-3, cob for cytochrome b, nad1-6 for NADH dehydrogenase subunits 1-6, $r r n L$ and $r r n S$ for large and small rRNA subunits. tRNA genes are indicated with their one-letter corresponding amino acids; the two tRNA genes for leucine and serine have different anticodons: L1 (anticodon TAG), L2 (TAA), S1 (TCT) and S2 (TGA). The GC content is plotted using a black sliding window, as the deviation from the average GC content of the entire sequence. GCskew is plotted as the deviation from the average GC-skew of the entire sequence. The inner cycle indicates the location of genes in the mitochondrial genome.

and Phthiraptera have evolved $134 \mathrm{Ma}$ and the sucking lice diversified in the Late Cretaceous, approximately $77 \mathrm{Ma}$. Moreover, the Anoplura and Rhynchophthirina have evolved 94 Ma (Fig. 6 and further details are shown in Fig. S2).

\section{Discussion}

The unidentified genes of the Psocodea. Mt genomes of bilaterians typically contain two rRNA, 22 tRNA, and 13 protein-coding genes (PCGs) and a control region on a single circular chromosome, with $\sim 16 \mathrm{~kb}$ in size ${ }^{16,17}$. This typical $\mathrm{mt}$ genome organization is conserved among most of the bilateral animals known from worms to insects, fish, and humans ${ }^{18-21}$. On the other hand, however, deviation from the typical mt genome organization has occurred in many bilateral animals. For instance, most nematodes lose atp 8 gene and thus only have 36 mitochondrial genes ${ }^{22,23}$, as well as in a tree frog species in which the nad5 gene is apparently missing ${ }^{24}$. So far, the reported species of the Psocodea, some PCG have not been identified in L. entomophila, L. sculptilis, Pthirus pubis, and Haematomyzus elephantis, i.e., nad4 and nad2 have not been found in the pubic louse and elephant louse, respectively ${ }^{5,6}$. Additional, nad4L has not been found in L. entomophila ${ }^{1}$. Intriguingly, we also did not find nad $4 L$ in L. sculptilis. However, nad $4 L$ is present in the other three species of Liposcelis (L. bostrychophila, $L$. decolor, and $L$. paeta $)^{1,8,10}$. There are many possibilities for lacking mitochondrial genes. Firstly, due to sequencing technology and sequencing method, a mini-chromosome contained nad4L (or nad4 or nad2) gene, which is not 


\begin{tabular}{|c|c|c|c|c|}
\hline Gene & Primer & Primer sequence $\left(5^{\prime}-3^{\prime}\right)$ & $\operatorname{Tm}\left({ }^{\circ} \mathrm{C}\right)$ & Amplicon size (bp) \\
\hline $\cos 1$ & UEA3 & TATAGCATTCCCACGAATAAATAA & \multirow{2}{*}{58} & \multirow{2}{*}{ UEA3-UEA8:1016 } \\
\hline $\operatorname{cox} 1$ & UEA8 & AAAAATGTTGAGGGAAAAATGTTA & & \\
\hline$c o b$ & CBF1 & TATGTACTACCATGAGGACAAATATC & \multirow{2}{*}{55} & \multirow{2}{*}{ CBF1-CBR1: 481} \\
\hline$c o b$ & CBR1 & ATTACACCTCCTAATTTATTAGGAAT & & \\
\hline$r r n L$ & 16Sar & CGCCTGTTTAACAAAAACAT & \multirow{2}{*}{51} & \multirow{2}{*}{ 16Sar-16Sbr: 513} \\
\hline$r r n L$ & $16 \mathrm{Sbr}$ & CCGGTCTGAACTCAGATCACGT & & \\
\hline nad5 & N5-F100 & GCTATAGCTGCTCCCACCCC & \multirow{2}{*}{60} & \multirow{2}{*}{ N5-F100-N5-R100: 311} \\
\hline nad5 & N5-R100 & ATAAATAAAAGAGCCTTGAATAAAGC & & \\
\hline $\cos 1$ & S1 & ATCCAATTCTATTTCAACACCT & \multirow{2}{*}{62} & \multirow{2}{*}{ S1-S2:1987 } \\
\hline nad5 & S2 & ATTCATCAACTCTTGTAACAGCCG & & \\
\hline nad5 & S3 & CGGCTGTTACAAGAGTTGATGAAT & \multirow{2}{*}{62} & \multirow{2}{*}{ S3-S4: 4934} \\
\hline$r r n L$ & S4 & TCTTAGGGTCTTCTCGTCTTTTTAT & & \\
\hline$r r n L$ & S5 & ATGGGTGGATGCCTTCTAATCTTTA & \multirow{2}{*}{56} & \multirow{2}{*}{ S5-S6: 4387} \\
\hline$c o b$ & S6 & ATGTTAGGAACGGTTCAAGAG & & \\
\hline$c o b$ & S7 & TAGTCTTAGCCCTACCATCAA & \multirow{2}{*}{57} & \multirow{2}{*}{ S7-S8: 3344} \\
\hline $\operatorname{cox} 1$ & S8 & AAAATATACACTTCAGGATGACCGA & & \\
\hline$c o b$ & S9 & AGCCAACTATCATACGGTTTTTTTC & \multirow{2}{*}{61} & \multirow{2}{*}{ S9-S10: 9005} \\
\hline$r r n L$ & S10 & AAACTCGGCAAAATTATGAAGCA & & \\
\hline$r r n L$ & S11 & СTACCCTGCTCTCTGATTTCAGTTT & \multirow{2}{*}{60} & \multirow{2}{*}{ S11-S12: 5631} \\
\hline$c o b$ & S12 & CTTTTGAGGGGCTACAGTGATTAC & & \\
\hline
\end{tabular}

Table 1. PCR primers used for amplification of the mitochondrial genome of Liposcelis sculptilis.

identified in L. sculptilis and L. entomophila (or Pthirus pubis and Haematomyzus elephantis). For example, the mt genome architecture in some thrips exhibit extreme chromosome size asymmetry that only nad6 and tRNA-Cys are on the $0.92 \mathrm{~kb}$ mini-circle chromosome $\mathrm{e}^{25}$. Secondly, these missing genes are functionally replaced by nuclear genes or normally transferred to the nuclear genome. For instance, $\mathrm{mt} \operatorname{cox} 2$ introgression into the nuclear genome has been reported ${ }^{26}$. Thirdly, the lacking genes have been lost in the long process of evolution. In fact, as some protein-coding genes of $\mathrm{mt}$ genes are essential in $\mathrm{mt}$ respiration and adenosine triphosphate production ${ }^{27}$, the loss of these genes would present serious metabolic challenges to cells. We inferred that the lacking of nad $4 L$ gene in L. sculptilis, more likely due to the first or the second possibilities.

For the unidentified tRNA genes in the Liposcelis, eight tRNA genes have not been found in mt genome of L. entomophila or L. paeta. Additionally, two of 22 typical tRNA genes were also not found in L. bostrychophila ${ }^{1,8}$, and one tRNA was unidentified in the L. sculptilis. Lacking of tRNA genes is more diverse, and, for example, has been reported in one gekkonid ${ }^{28}$, in one caecilian amphibian ${ }^{29}$, and in isopod crustacean ${ }^{30}$. Moreover, among lower metazoans, a massive loss of tRNA was reported in cnidarians ${ }^{31}$. Altogether, it is unclear whether in these cases the gene loss is due to gene transfer to the nuclear genome or reflects loss of the protein function ${ }^{32}$.

The mitochondrial genome strand asymmetry and structure of the Liposcelis. Strand asymmetry is reflected by AT skew and GC skew. Positive AT skew values indicate more A than T on the target strand, and positive GC skew values indicate more G than C, and vice versa ${ }^{33}$. In terms of $L$. sculptilis, the A $+\mathrm{T}$ content is $76.5 \%$, which was lower than the L. entomophila (78.6\%) in Liposcelis. The AT skew is 0.018 and the CG skew is 0.047 , and these results indicated a low degree of strand asymmetry of the base composition in the L. sculptilis $\mathrm{mt}$ genome. Generally, insect mt genomes, in terms of the structure, gene order, gene content, are very stable, however, this rule does not apply in the genus of Liposcelis. From the results of our studies in five Liposcelis species, the tremendous differences in these features were existed (Figs 3 and 4). Such differences among mt genomes, were first discovered within a genus in insects. Even some of the gene blocks are stable within a genus of Anoplura, which have highly fragmented $\mathrm{mt}$ genomes and violently gene rearrangement. For instance, the sucking lice, Pediculus humanus and P. capitis, have fragmented mt genomes with 20 minichromosomes, but the gene order of protein-coding genes and gene content, were identical across the corresponding minichromosomes ${ }^{4,5}$. The same example also appeared between the pig lice, Haematopinus apri and $H$. suis ${ }^{34}$. The phylogenetic relationships between booklice and Phthiraptera are close from gene rearrangement. However, three barklices (Lepidopsocid sp., Psococerastis albimaculata, and Longivalvus hyalospilus), have relatively conservative gene order ${ }^{12,35}$, also belong to Psocoptera. They shared lots of gene blocks. For the convenience and accuracy of communication, our previous studies have introduced the concept of "mitochondrial karyotype" or "mitochondrial genome karyotypes" for describing the violent variation of $\mathrm{mt}$ genomes in sucking lice and booklice ${ }^{6,36}$.

Phylogenetic relationships among major lineages of the Liposcelis inferred from mitochondrial genome sequences. The phylogenetic tree showed a close relationship between the booklice (Liposcelis) and parasitic lice (sucking lice). Therefore, the order Psocoptera was paraphyletic, and this result was consistent with previous studies ${ }^{13,17-19}$. Mt gene rearrangement has been substantially faster in the lineage leading to the 


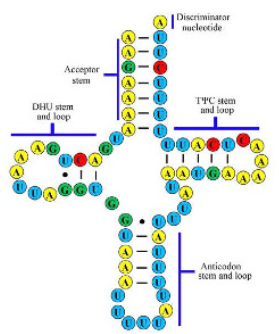

Asn(N)

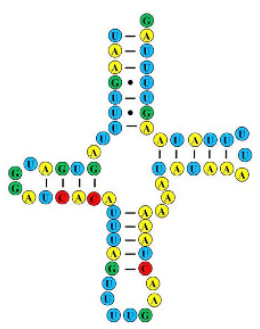

Gln(Q)

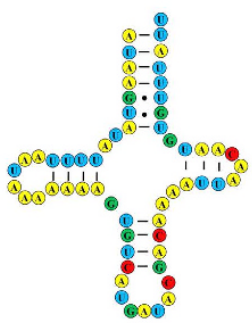

Ile(I)

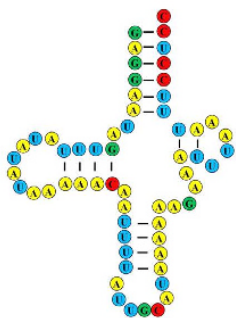

Ala (A)

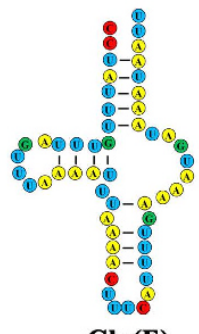

Glu(E)

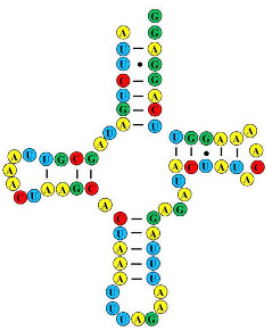

Leu1(L)(CUN)

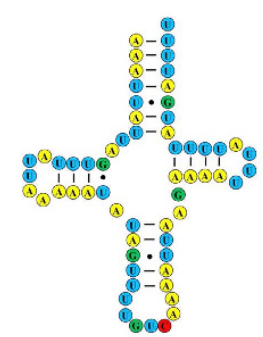

Asp(D)

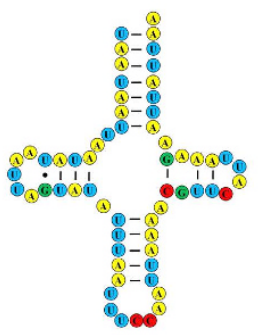

Gly(G)

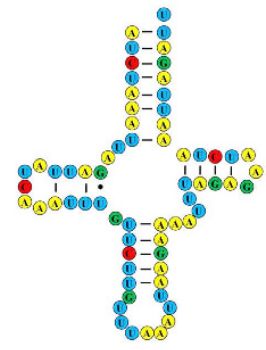

Leu2(L)(UUR)

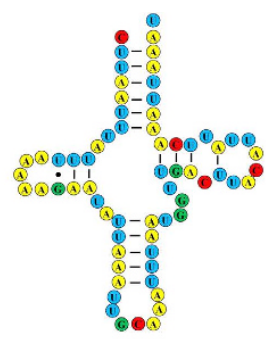

Cys(C)

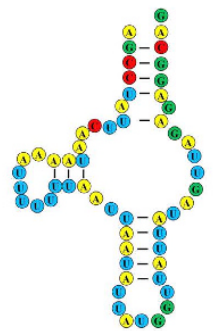

His(H)

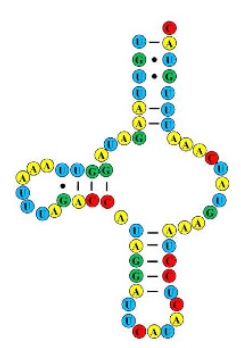

Met(M)

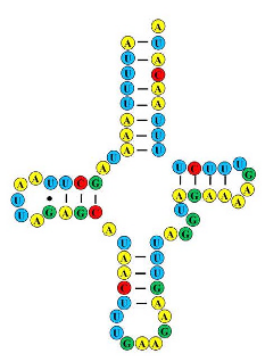

Phe(F)

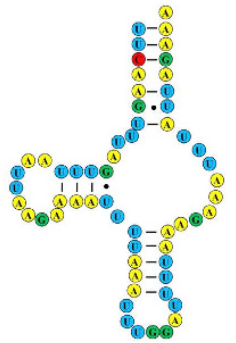

Pro(P)

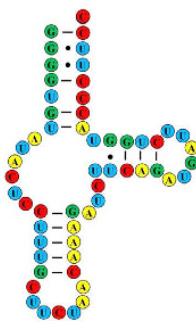

Ser1(S)(AGN)

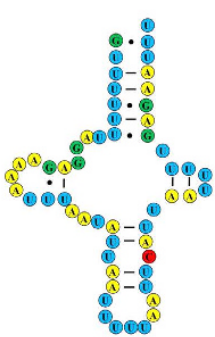

Lys(K)

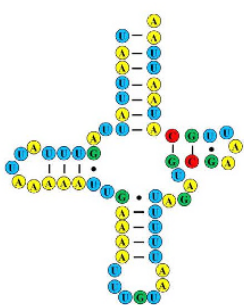

Thr(T)

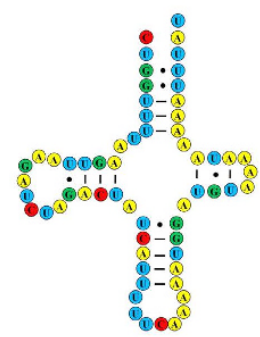

$\operatorname{Trp}(\mathbf{W})$

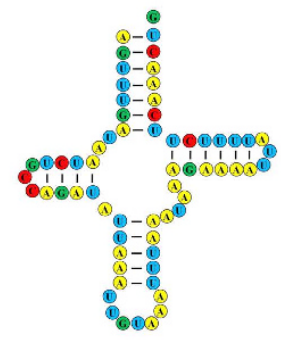

$\operatorname{Tyr}(\mathbf{Y})$

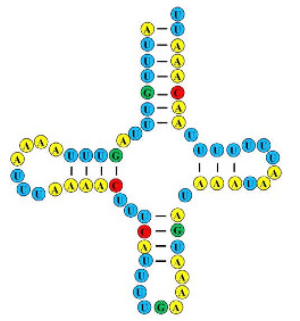

Ser2(S)(UCN)

Figure 2. Putative secondary structures of the 21 tRNA genes identified in the mitochondrial genome of L. sculptilis. Bars indicate Watson-Crick base pairings, and dots between G and U pairs mark canonical base pairings in RNA.

booklice and the parasitic lice than in the lineage leading to the barklice ${ }^{12}$. Lifestyle change of booklice appears to be associated with the contrasting rates in $\mathrm{mt}$ gene rearrangements between the two clades of the Psocodea ${ }^{37-39}$.

Within the genus Liposcelis, L. paeta and L. sculptilis formed a clade and then clustered with L. bostrychophila. These three species, from group $\mathrm{D}^{40}$, were most closely related to L. entomophila (group A). The current 

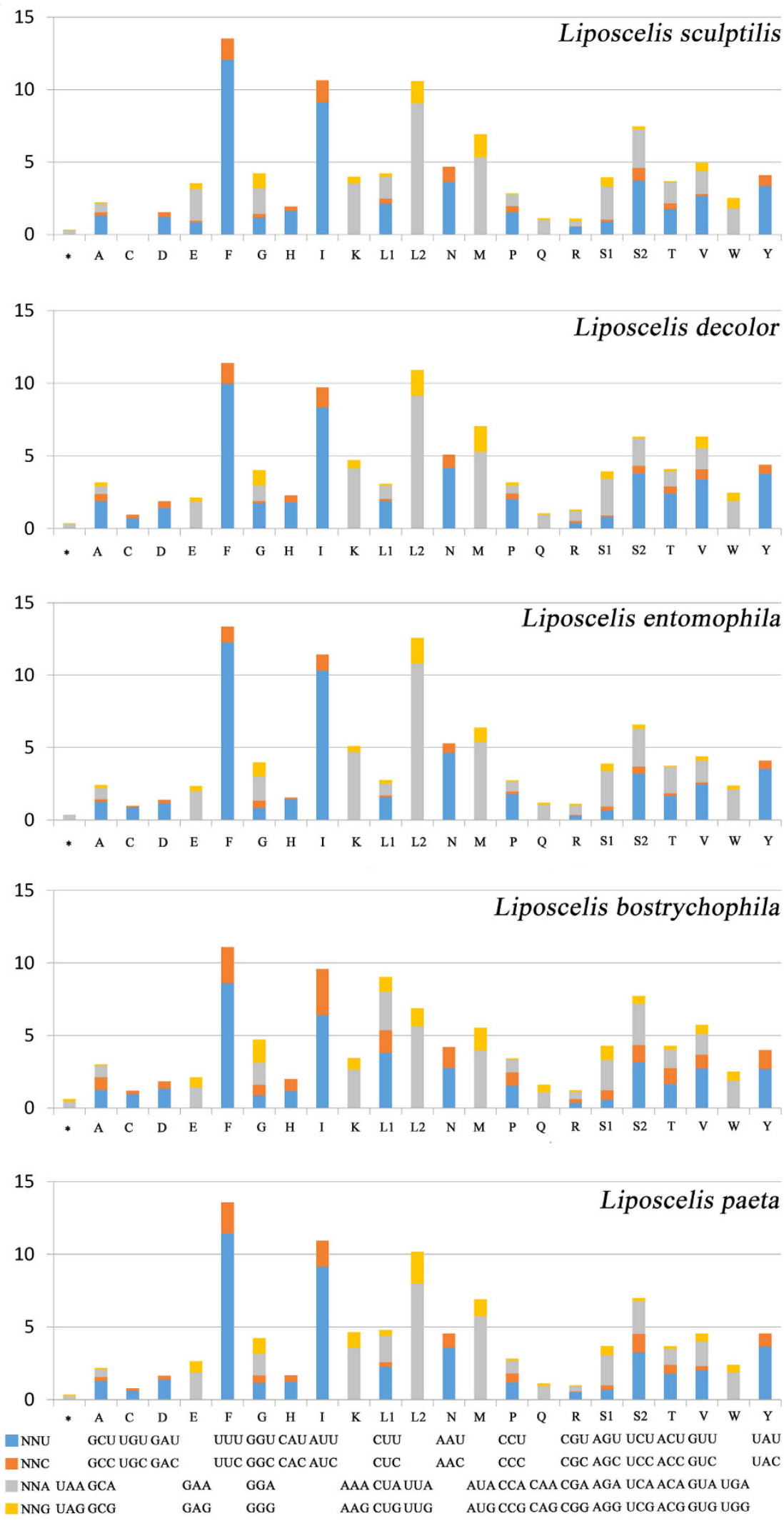

Figure 3. Relative synonymous codon usage (RSCU) for protein coding genes of five booklice.

Abbreviations of tRNA genes are according to the single letter according to the IPUC-IUB one-letter amino acid codes. 


\begin{tabular}{|c|c|c|c|c|c|}
\hline Gene & L. decolor & L. entomophila & L. paeta & L. bostrychophila & L. sculptilis \\
\hline atp6 & ATG/TAA & ATA/TAA & ATA/TAA & ATA/TAA & ATA/TAA \\
\hline atp8 & ATA/TAG & ATA/TAA & ATA/TAG & GTG/TAG & ATA/TAG \\
\hline$c o b$ & ATA/TAA & ATA/TAA & ATA/TAA & ATT/TAA & ATA/TAA \\
\hline $\operatorname{cox} 1$ & TTG/TAA & ATT/TAA & ATA/TAG & ATC/TAA & ATA/TAA \\
\hline $\cos 2$ & ATT/TAA & ATA/TAA & ATA/TAG & ATA/TAA & ATA/TAA \\
\hline $\cos 3$ & ATA/TAA & ATA/TAA & TTG/TAA & ATA/TAA & ATG/TAA \\
\hline nad1 & ATA/TAA & ATA/TAA & ATT/TAA & ATC/T & ATT/TAA \\
\hline nad2 & ATA/TAA & ATA/TAA & ATA/TAA & ATT/TAA & ATT/TAA \\
\hline nad3 & ATG/TAA & ATA/TAA & ATA/TAA & ATG/TAG & ATG/TAA \\
\hline nad4 & ATA/TAA & ATT/TAA & ATA/TAA & ATC/TAA & ATT/TAA \\
\hline nad5 & ATA/TAA & ATT/TAA & ATT/TAA & ATT/TAG & ATA/TAA \\
\hline nad6 & ATG/TAA & ATA/TAA & ATA/TAA & ATA/TAA & ATA/TAA \\
\hline nad4L & ATA/TAA & & ATA/T & ATT/TAA & \\
\hline
\end{tabular}

Table 2. The start and stop codons of protein coding gene for Liposcelis species.

Hypothetical ancestor of insect

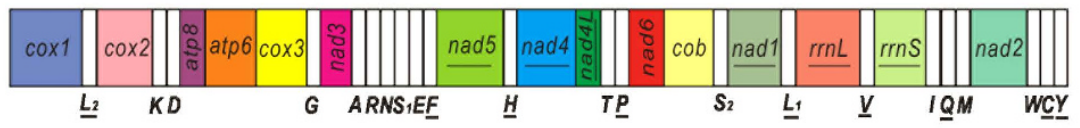

Lepidopsocid SP.(Psocoptera, Trogiomorpha)

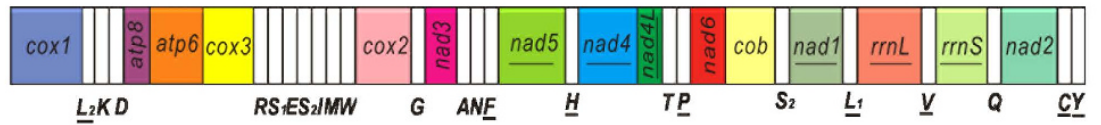

Liposcelis decolor (Psocoptera, Troctomorpha)

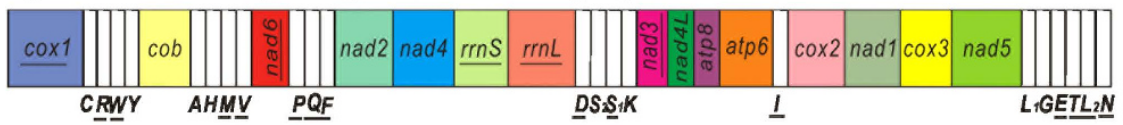

Liposcelis sculptilis (Psocoptera, Troctomorpha)

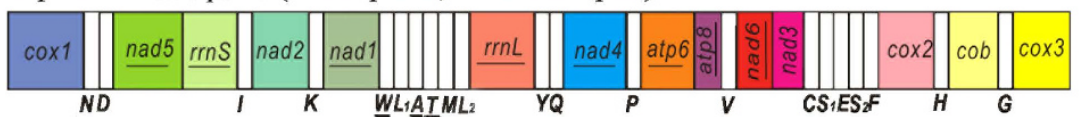

Liposcelis entomophila (Psocoptera, Troctomorpha)

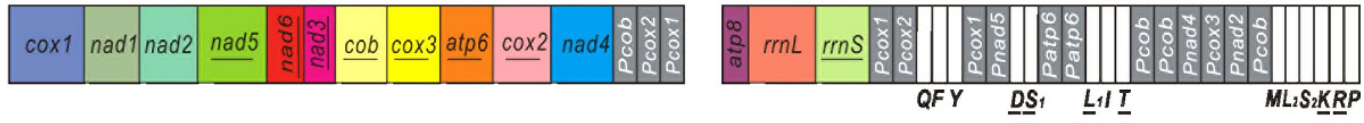

Liposcelis paeta (Psocoptera, Troctomorpha)
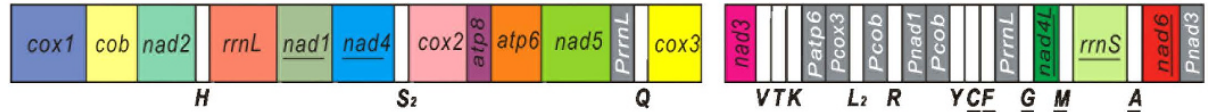

Liposcelis bostrychophila (Psocoptera, Troctomorpha)
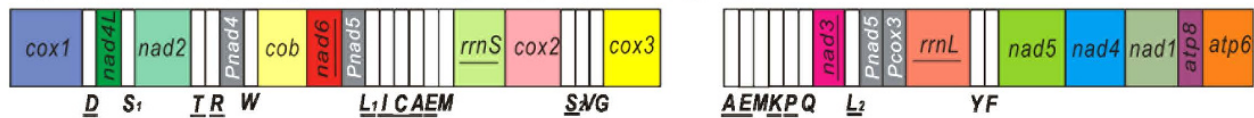

Figure 4. Arrangement of mitochondrial genes in Liposcelis and the hypothetical ancestor of the arthropods. Circular genomes have been arbitrarily linearized for ease of comparison. Gene names are the standard abbreviations used in the present study. tRNA genes are designated by the single letter according to the IPUC-IUB one-letter amino acid codes. Genes which are underlined are encoded on the opposite strand to the majority of genes in that particular genome. Gray, and white boxes represent pseudogenes, and transfer RNA genes, respectively. The boxes in 15 colors represent 13 protein coding genes and 2 ribosomal RNA genes. 


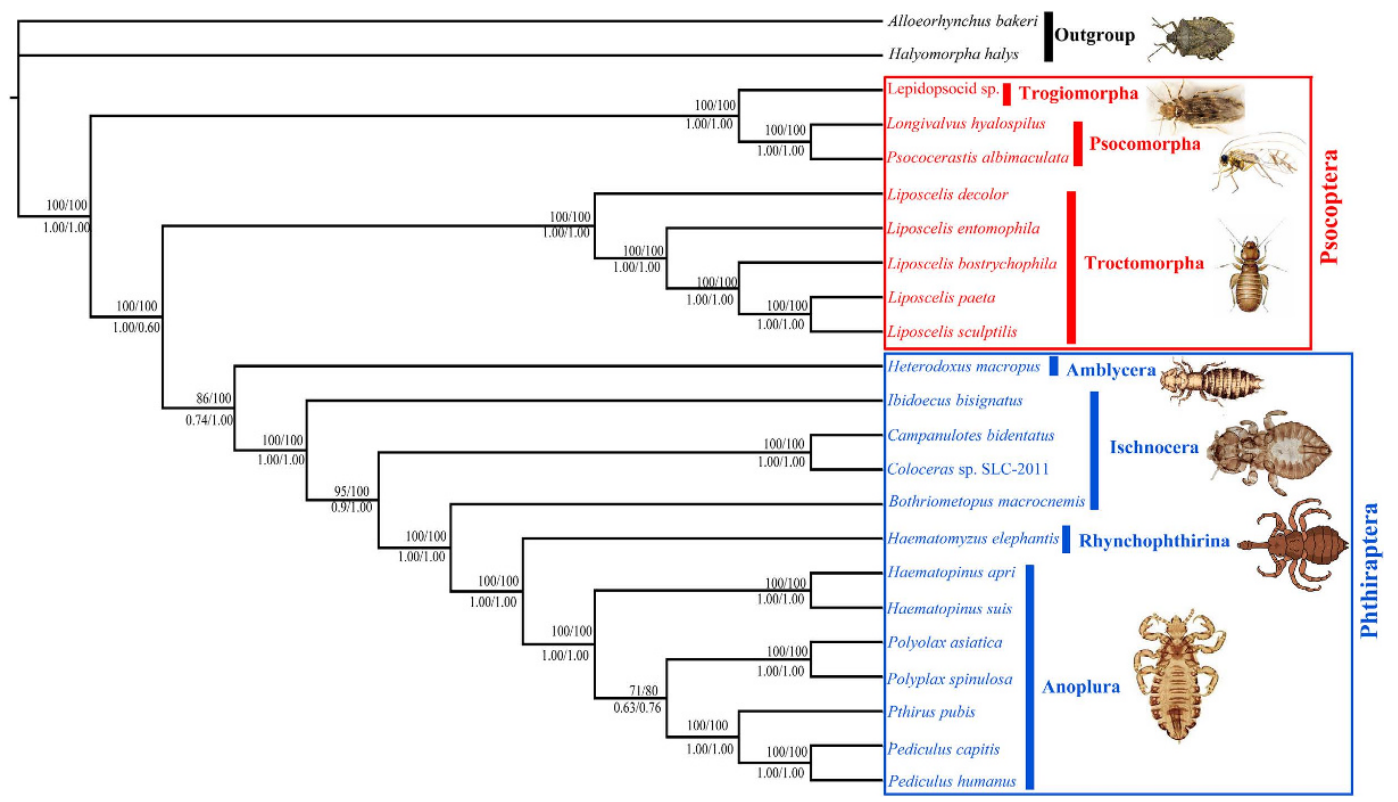

Figure 5. Phylogenetic relationships among major lineages of the Psocodea inferred from mitochondrial genome sequences. Numbers close to the branching points are ML bootstrap support values (above) and Bayesian posterior probabilities (below) in percentages; only support above $50 \%$ is shown. Numbers from left to right are from PCG123R and AA alignments respectively.

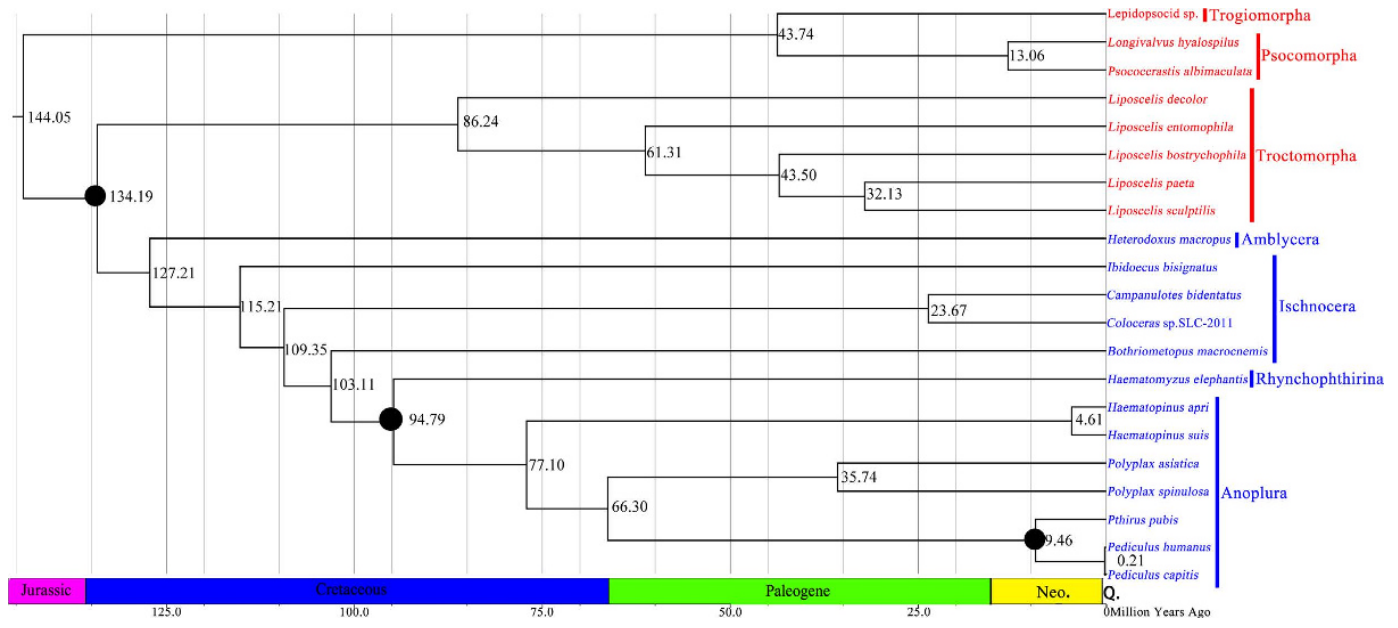

Figure 6. Chronogram for the Psocodea. Chronogram is the Bayesian topology resulting from analysis of the 11 genes (cox1, cox2, cox3, atp6, cob, nad1, nad3, nad5, nad6, rrnS and $r r n L$ ) data set in BEAST ${ }^{53}$. Divergence times were estimated using three calibrations (The potential ancient origin of lice considering the recently described 100-145 Ma book louse fossil; 94-101 Ma for the split between Rhynchophthirina and Anoplura; this ancestral to all human lice has been stable for at least $7 \mathrm{Ma}$ ), indicated by filled circles at nodes.

classification of genus Liposcelis included four groups (A, B, C, and D $)^{11,41}$, which have big difference in morphological, physiology and molecular biology aspects ${ }^{41,42}$. Although the five Liposcelis species are different from each other in mt genome organization, they were clustered together with strong support in the phylogenetic tree. Then, these results suggest unusually fast evolution in $\mathrm{mt}$ genome organization in the booklice of the genus Liposcelis.

Estimation of divergence times. Fossil calibrations for lice are lacking ${ }^{13,15}$. Based on a fossil calibration, previous study indicated that parasitic lice and booklice minimally have diverged $100 \mathrm{Ma}^{13}$. For a basal calibration representing the split between Rhynchophthirina and Anoplura, we chose the time of basal diversification in placental mammals in a previous study ${ }^{43}$, because we believed it reasonable that sucking lice could not have diversified until they had appropriate hosts to colonize ${ }^{43,44}$. It has been determined that time of basal diversification in placental mammals was 94-109 Ma; therefore, a calibration with a mean of 101 (and standard deviation of 3.5) was used to represent the basal split between the Rhychophthirina and Anoplura ${ }^{14,43}$. Additionally, some studies 
have documented the most recent common ancestor of human lice (Pediculus humanus, Pediculus capitis, and Pthirus pubis) has been stable for at least $7 \mathrm{Ma}^{5}$. Totally, these three calibration points were used in combination, as well as individually, to cross-check the other calibration points, in the divergence dating analyses.

Our analyses indicated that the most recent common ancestor of Liposcelididae and Phthiraptera has been diversified in Late Jurassic to Early Cretaceous, approximately $134 \mathrm{Ma}$ (Fig. 6). The estimated divergence time of Liposcelididae and Phthiraptera was largely consistent with a recent estimates ${ }^{45}$. Moreover, the results, sucking lice diversified in the Late Cretaceous, approximately $77 \mathrm{Ma}$; Anoplura and Rhynchophthirina have evolved $94 \mathrm{Ma}$ (Fig. 6), were also consistent with a previous study ${ }^{14}$. However, another previous study reported that Liposcelididae and Amblycera were sister group and the Ischnocera was monophly, was inconsistent with the most recent research ${ }^{1,8,10,12,15}$.

\section{Materials and Methods}

Sample collection, DNA extraction and amplification of mitochondrial genome. L. sculptilis was collected at a grain storage in Gansu Province, China, and identified according to their morphological characteristics $^{11,44}$. Total genomic DNA was extracted from $\sim 300$ booklouse specimens $(20 \mathrm{mg})$ using a TIANamp Genomic DNA Kit (Tiangen Biotech, Beijing, China) and stored at $-20^{\circ} \mathrm{C}$. Partial sequences of cox $1, \operatorname{cob}$, nad5, and $r r n L$ of $L$. sculptilis were amplified initially by PCR with primer pairs UEA3-UEA8 (1016bp), CBF1-CBR1 (481 bp), N5-F100-N5-R100 (311 bp) and 16Sar-16Sbr (513 bp), respectively (Table 1). Four pairs of primers, S1-S2, S3-S4, S5-S6 and S7-S8, were designed from cox1, cob, nad5, and $r r n L$ (Table 1). Four overlapping fragments were amplified by long PCR with S1-S2, S3-S4, S5-S6 and S7-S8, sequenced and assembled into contigs with SeqMan (DNAStar). To verify $L$. sculptilis chromosome and avoid the mistake might be caused by primers (S9-S10 and S11-S12) at cob and rrnL, a 9,005 bp fragment and a 5,631 bp fragment were amplified additionally with primers S9-S10 and S11-S12 (Table 1).

LA Taq ( $5 \mathrm{U} / \mu \mathrm{L}$, Takara) was used in long PCRs to amplify overlapping fragments. Each long PCR reaction is $25 \mu \mathrm{L}$ in volume, containing $1.0 \mu \mathrm{L}$ each of forward primer $(10 \mu \mathrm{M})$ and reverse primer $(10 \mu \mathrm{M}), 4.0 \mu \mathrm{L}$ of dNTPs mix (each $2.5 \mathrm{mM}), 1.0 \mu \mathrm{L}$ of template DNA, $2.5 \mu \mathrm{L} \mathrm{MgCl}_{2}(25 \mathrm{mM}), 2.5 \mu \mathrm{L}$ of $10 \times$ LA PCR reaction buffer II, $12.75 \mu \mathrm{L}$ ddH2O and $0.25 \mu \mathrm{L}$ LA Taq DNA polymerase $(5 \mathrm{U} / \mu \mathrm{L}$, Takara). All reactions were carried out using $\mathrm{C} 1000^{\mathrm{TM}}$ thermal cyclers (Bio-RAD, Hercules, CA, USA) with the following conditions: $2 \mathrm{~min}$ denaturation at $94^{\circ} \mathrm{C}, 37$ cycles of $94^{\circ} \mathrm{C}$ for $20 \mathrm{~s}, 58^{\circ} \mathrm{C}$ for $50 \mathrm{~s}, 68^{\circ} \mathrm{C}$ for $5-10 \mathrm{~min}$ (depending on target size, $1 \mathrm{~min} / \mathrm{kb}$ ), followed by a final extension at $68^{\circ} \mathrm{C}$ for $15 \mathrm{~min}$. Positive and negative controls were always executed with each PCR experiment to detect DNA contamination and false positive amplifications. PCR products were checked by agarose gel (1\%) electrophoresis. Gel-purified amplification products $<3 \mathrm{~kb}$ in size were ligated into pGEM-T Easy vectors (Promega), and introduced into Escherichia coli (DH5 $\alpha$, Beijing TransGen Biotech, Beijing, China). Followed by ampicillin selection, plasmid DNAs from positive clones were sequenced with M13 primers. Longer PCR products $(>3 \mathrm{~kb}$ ) were directly sequenced with both forward and reverse PCR primers and internal primers by primer walking. All products were sequenced by Life Technologies in Guangzhou, China.

Sequence assembly, annotation and analysis. SeqMan (DNAStar) was used to assemble the four overlapping nucleotide sequences, which were further confirmed by manually inspection. The protein-coding and rRNA genes were identified using the program ORF Finder (http://www.ncbi.nlm.nih.gov/gorf/gorf.html) and BLAST searches against the GenBank database, respectively. Subsequently, all of these genes were further confirmed by alignment with homologous genes from those of other booklice and lice species. The transfer RNA genes were identified by their cloverleaf secondary structure using ARWEN ${ }^{46}$ with default parameters and tRNAscan-SE $1.21^{47}$ with Search Mode = EufindtRNA-Cove, Genetic Code = Invertebrate Mito and Cove score cutoff $=0.1$. The base composition was analyzed with MEGA $5^{48}$. Sequences of mt genomes of other booklice and lice were retrieved from GenBank (Table S1).

Sequence alignment and phylogenetic analysis. Eight species from the Psocoptera and thirteen species from the Phthiraptera were included in our phylogenetic analysis (Table S1). Two true bugs Alloeorhynchus bakeri and Halyomorpha halys were used as outgroups ${ }^{12}$.

Sequences of all mt protein-coding genes and rRNA genes except nad4, nad4L, nad2, atp 8 were used in phylogenetic analysis. nad $4 L$ and atp 8 were excluded because they are too short to align among the Psocodean species. nad4 and nad2 was excluded because it was not identified in the human pubic louse, Pthirus pubis ${ }^{5}$ or in the elephant louse, Haematomyzus elephantis ${ }^{6}$. Two alignments were used for phylogenetic analyses: 1) a concatenated nucleotide sequence alignment of nine protein-coding genes and two rRNA genes; 2) a concatenated amino acid sequence alignment of nine protein-coding genes. Nucleotide sequences of all protein-coding genes and rRNA genes were aligned using the default settings in ClustalW as implemented in MEGA $5^{48}$. Amino acid sequences of PCGs were also aligned in ClustalW. All of the alignments were then imported into the Gblocks server (http://molevol.cmima.csic.es/castresana/Gblocks_server.html) to remove poorly aligned sites ${ }^{49}$. Gblocks server was applied with the 'codons', 'DNA' and 'protein' mode for PCG nucleotide sequences, rRNA sequences and PCG amino acid sequences, respectively, and with all options for a stringent selection were chosen.

Subsequent analyses were performed on the combined dataset using Maximum likelihood (ML) and Bayesian inference (BI). BI was performed using MrBayes 3.2 $2^{50}$ and ML was performed using RAxML 7.7.1 $1^{51}$. For ML, the GTRGAMMA model was selected for the concatenated datasets, with 1000 bootstrap replicates. For BI, the best-fitting nucleotides models were chosen using PartitionFinder V1.1.1 $1^{52}$ as follows: TIM $+\mathrm{I}+\mathrm{G}$ : cox1; GTR + I + G: atp6, cob, cox2, cox3, nad1, nad3, nad5; HKY + I + G: nad6; TVM + I + G: rrnL, rrnS; the best-fitting amino acids models were chosen as follows: MtArt + I+ G: $\operatorname{cox} 1$; MtArt + I+ G + F: $\operatorname{cox} 2, \operatorname{cox} 3, \operatorname{cob}$, atp6, nad1, nad3, nad5, and nad6. Two independent sets of Markov chains were run, each with one cold and three 
heated chains for $1 \times 10^{7}$ generations, and every 1000th generation was sampled. Convergence was inferred when a standard deviation of split frequencies $<0.01$ was completed. Sump and sumt burninfrac was set to $25 \%$ and contype was set to allcompat.

Divergence dating analysis. We performed divergence date analyses based on the combined $11 \mathrm{mt}$ genes dataset of Psocodean (Table S1). The molecular clock was calibrated using three minimum age constraints based on one fossil and two conclusions (100-145 Ma for the split between lice and Liposcelididae, 94-101 Ma for the split between Rhynchophthirina and Anoplura, and the ancestor of three human lice has been stable for at least $7 \mathrm{Ma})^{5,13,14}$. Analyses were performed using a relaxed molecular clock model in the Bayesian phylogenetic software BEAST 1.8.0 $0^{53}$. Rate variation was modeled among branches using uncorrelated lognormal relaxed clocks ${ }^{53}$. A Yule speciation process was used for the tree prior and posterior distributions of parameters, including the tree, were estimated using MCMC sampling ${ }^{54}$. We performed two replicate MCMC runs, with the tree and parameter values sampled every 5000 steps over a total of 50 million generations. A maximum clade credibility tree was obtained using Tree Annotator within the BEAST software package with a burn-in of 1000 trees. Acceptable sample sizes and convergence to the stationary distribution were checked using Tracer $1.5^{53}$.

\section{References}

1. Chen, S. C. et al. Evolution of multipartite mitochondrial genomes in the booklice of the genus Liposcelis (Psocoptera). BMC Genomics 15, 861 (2014).

2. Adi, B., Mccormick, L. J. \& Perlman, S. J. Rickettsia felis infection in a common household insect pest, Liposcelis bostrychophila (Psocoptera: Liposcelidae). Appl. Environ. Microbiol. 76, 2280-2285 (2010).

3. Yang, Q. et al. Rapid molecular diagnosis of the stored-product psocid Liposcelis corrodens (Psocodea: Liposcelididae): speciesspecific PCR primers of 16S rDNA and COI. J. Stored Prod. Res. 54, 1-7 (2013).

4. Shao, R., Kirkness, E. F. \& Barker, S. C. The single mitochondrial chromosome typical of animals has evolved into 18 minichromosomes in the human body louse, Pediculus humanus. Genome Res. 19, 904-912 (2009).

5. Shao, R., Zhu, X. Q., Barker, S. C. \& Herd, K. Evolution of extensively fragmented mitochondrial genomes in the lice of humans. Genome Biol. Evol. 4, 1088-1101 (2012).

6. Shao, R. et al. Fragmented mitochondrial genomes in two suborders of parasitic lice of eutherian mammals (Anoplura and Rhynchophthirina, Insecta). Sci. Rep. 5 (2015).

7. Cameron, S. L., Yoshizawa, K., Mizukoshi, A., Whiting, M. F. \& Johnson, K. P. Mitochondrial genome deletions and minicircles are common in lice (Insecta: Phthiraptera). BMC Genomics 12, 1-15 (2011).

8. Wei, D. D. et al. The multipartite mitochondrial genome of Liposcelis bostrychophila: insights into the evolution of mitochondrial genomes in bilateral animals. PLoS ONE 7, e33973 (2012).

9. Perlman, S. J., Hodson, C. N., Hamilton, P. T., Opit, G. P. \& Gowen, B. E. Maternal transmission, sex ratio distortion, and mitochondria. Proc. Natl. Acad. Sci. USA 112, 10162-10168 (2015).

10. Chen, S. C., Wei, D. D., Shao, R., Dou, W. \& Wang, J. J. The complete mitochondrial genome of the booklouse, Liposcelis decolor: insights into gene arrangement and genome organization within the genus Liposcelis. PLoS ONE 9, e91902 (2014).

11. Li, F. S. Psocoptera of China. Beijing, Science Press 77-102 (2002).

12. Li, H. et al. Mitochondrial genomes of two barklice, Psococerastis albimaculata and Longivalvus hyalospilus (Psocoptera: Psocomorpha): Contrasting rates in mitochondrial gene rearrangement between major lineages of Psocodea. PLoS ONE 8, e61685 (2013).

13. Grimaldi, D. \& Engel, M. S. Fossil Liposcelididae and the lice ages (Insecta: Psocodea). Proc. Roy. Soc. B-Biol. Sci. 273, 625-633 (2006).

14. Light, J. E., Smith, V. S., Allen, J. M., Durden, L. A. \& Reed, D. L. Evolutionary history of mammalian sucking lice (Phthiraptera: Anoplura). BMC Evol. Biol. 10, 292 (2010).

15. Smith, V. S. et al. Multiple lineages of lice pass through the K-Pg boundary. Biol. Letters 7, 782-785 (2011).

16. Boore, J. L. Animal mitochondrial genomes. Nucleic Acids Res. 27, 1767-1780 (1999).

17. Lavrov, D. V. Key transitions in animal evolution: a mitochondrial DNA perspective. Integr. Comp. Biol. 47, 734-743 (2007).

18. Anderson, S. et al. Sequence and organization of the mitochondrial human genome. Nature 290, 457-465 (1981).

19. Lewis, O. L., Farr, C. L. \& Kaguni, L. S. Drosophila melanogaster mitochondrial DNA: completion of the nucleotide sequence and evolutionary comparisons. Insect Mol. Biol. 4, 263-278 (1995).

20. Cao, Y., Waddell, P. J., Okada, N. \& Hasegawa, M. The complete mitochondrial DNA sequence of the shark Mustelus manazo: evaluating rooting contradictions to living bony vertebrates. Mol. Biol. Evol. 15, 1637-1646 (1998).

21. Lavrov, D. V. \& Brown, W. M. Trichinella spiralis mtDNA: a nematode mitochondrial genome that encodes a putative ATP8 and normally structured tRNAS and has a gene arrangement relatable to those of coelomate metazoans. Genetics 157, 621-637 (2001)

22. Gissi, C., Iannelli, F. \& Pesole, G. Evolution of the mitochondrial genome of Metazoa as exemplified by comparison of congeneric species. Heredity 101, 301-320 (2008).

23. Sophie, B., Stewart, D. T. \& Hoeh, W. R. Characterization of a mitochondrial ORF from the gender-associated mtDNAs of Mytilus spp. (Bivalvia: Mytilidae): identification of the "missing” ATPase 8 gene. Mar. Genomics 3, 11-18 (2010).

24. Zhang, P. et al. The complete mitochondrial genome of a tree frog, Polypedates megacephalus (Amphibia: Anura: Rhacophoridae), and a novel gene organization in living amphibians. Gene 346, 133-143 (2005).

25. Dickey, A. M. et al. A novel mitochondrial genome architecture in thrips (Insecta: Thysanoptera): extreme size asymmetry among chromosomes and possible recent control region duplication. BMC Genomics 16, 1-7 (2015).

26. Chung, W. K. \& Steiper, M. E. Mitochondrial COII introgression into the nuclear genome of Gorilla gorilla. Int. J. Primatol. 29, 1341-1353 (2008).

27. Wolstenholme, D. R. Animal mitochondrial DNA: structure and evolution. Int. Rev. Cytol. 141, 173-216 (1992).

28. Kumazawa, Y., Miura, S., Yamada, C. \& Hashiguchi, Y. Gene rearrangements in gekkonid mitochondrial genomes with shuffling, loss, and reassignment of tRNA genes. BMC Genomics 15, 1-13 (2014).

29. San Mauro, D., Gower, D., Oommen, O. M. \& Zardoya, R. Phylogeny of caecilian amphibians (Gymnophiona) based on complete mitochondrial genomes and nuclear RAG1. Mol. Phylogenet. Evol. 33, 413-427 (2004).

30. Kilpert, F. \& Podsiadlowski, L. The complete mitochondrial genome of the common sea slater, Ligia oceanica (Crustacea, Isopoda) bears a novel gene order and unusual control region features. BMC Genomics 7, 1-18 (2006).

31. Mónica, M., Collins, A. G., Takaoka, T. L., Kuehl, J. V. \& Boore, J. L. Naked corals: skeleton loss in Scleractinia. Proc. Natl. Acad. Sci. USA 103, 9096-9100 (2006)

32. Adams, K. L. \& Palmer, J. D. Evolution of mitochondrial gene content: gene loss and transfer to the nucleus. Mol. Biol. Evol. 29, 380-395 (2003)

33. Wei, S. J. et al. New views on strand asymmetry in insect mitochondrial genomes. PLoS ONE 5, e12708 (2010). 
34. Jiang, H., Barker, S. C. \& Shao, R. Substantial variation in the extent of mitochondrial genome fragmentation among blood-sucking lice of mammals. Genome Biol. Evol. 5, 1298-1308 (2013).

35. Shao, R., Campbell, N. J., Schmidt, E. R. \& Barker, S. C. Increased rate of gene rearrangement in the mitochondrial genomes of three orders of hemipteroid insects. Mol. Biol. Evol. 18, 1828-1832 (2001).

36. Wei, D. D., Shao, R. F., Chen, S. C. \& Wang, J. J. Progress in mitochondrial genome diversity and phylogeny of Psocodea. Acta Entomol. Sin. 57, 483-494 (2014).

37. Johnson, K. P., Yoshizawa, K. \& Smith, V. S. Multiple origins of parasitism in lice. Proc. Roy. Soc. B-Biol. Sci. 271, 1771-1776 (2004).

38. Hopkins, He. G. The host-associations of the lice of mammals. Proc. Zool. Soc. Lond. 119, 387-604 (1949).

39. Mockford, E. L. Some Psocoptera from the plumage of birds. Proc. Entomol. Soc. Wash. 69, 307-309 (1967)

40. Lienhard, C. Revision of the western Palaearctic species of Liposcelis Motschulsky (Psocoptera: Liposcelididae). Zool. J. Abt. 117-174 (1990).

41. Wei, D. D. et al. Sequence analysis of the ribosomal internal transcribed spacers region in psocids (Psocoptera: Liposcelididae) for phylogenetic inference and species discrimination. J. Econ. Entomol. 104, 1720-1729 (2011).

42. Cheng, W. X., Dou, W., Chai, Y. X. \& Wang, J. J. Comparison of biochemical and toxicological characterizations of glutathione Stransferases and superoxide dismutase between Liposcelis bostrychophila Badonnel and L. entomophila (Enderlein) (Psocoptera: Liposcelididae). Pestic. Biochem. Physiol. 89, 151-157 (2007).

43. Bininda-Emonds, O. R. P. et al. The delayed rise of present-day mammals. Nature 446, 507-512 (2007).

44. Lyal, C. H. C. Phylogeny and classification of the Psocodea, with particular reference to the lice (Psocodea: Phthiraptera). Syst. Entomol. 10, 145-165 (1985).

45. Bernhard, M. et al. Phylogenomics resolves the timing and pattern of insect evolution. Science 346, 763-767 (2014).

46. Laslett, D. \& Canback, B. ARWEN: a program to detect tRNA genes in metazoan mitochondrial nucleotide sequences. Bioinformatics 24, 172-175 (2008).

47. Lowe, T. M. \& Eddy, S. R. A program for improved detection of transfer RNA genes in genomic sequence. Nucleic Acids Res. 25, 955-964 (1997).

48. Tamura, K. et al. MEGA5: molecular evolutionary genetics analysis using maximum likelihood, evolutionary distance and maximum parsimony methods. Mol. Biol. Evol. 28, 2731-2739 (2011).

49. Castresana, J. Selection of conserved blocks from multiple alignments for their use in phylogenetic analysis. Mol. Biol. Evol. 17, $540-552(2000)$.

50. Ronquist, F. et al. MrBayes 3.2: efficient Bayesian phylogenetic inference and model choice across a large model space. Syst. Biol. 61, 539-542. (2012).

51. Stamatakis, A., Hoover, P. \& Rougemont, J. A rapid bootstrap algorithm for the RAxML web servers. Syst. Biol. 57, 758-771 (2008).

52. Allery, C., Beghein, C. \& Hamdouni, A. Partitionfinder: combined selection of partitioning schemes and substitution models for phylogenetic analyses. Mol. Biol. Evol. 29, 1695-1701 (2012).

53. Drummond, A. J. \& Rambaut, A. BEAST: Bayesian evolutionary analysis by sampling trees. BMC Evol. Biol. 7, 214 (2007).

54. Gernhard, T. The conditioned reconstructed process. J. Theor. Biol. 253, 769-778 (2008).

55. Stothard, P. \& Wishart, D. S. Circular genome visualization and exploration using CGView. Bioinformatics 21, 537-539 (2005).

\section{Acknowledgements}

This study was supported by the National Natural Sciences Foundation (31301667), the Fundamental and Advanced Research Projects of Chongqing (cstc2015jcyjA80009), and the Fundamental Research Funds for the Central Universities, China (XDJK2015B034; 2362015xk04). We thank Yan-Li Che and Qi-Kun Bai for assistance.

\section{Author Contributions}

Y.S., D.-D.W. and J.-J.W. conceived and designed the experiments. Y.S., Q.C. and Y.-J.Q. performed the experiments. Y.S., D.-D.W., F.S., D.W. and J.-J.W. analyzed the data. Y.S., D.-D.W. and J.-J.W. wrote the paper, and all authors reviewed the manuscript.

\section{Additional Information \\ Supplementary information accompanies this paper at http://www.nature.com/srep}

Competing financial interests: The authors declare no competing financial interests.

How to cite this article: Shi, Y. et al. The mitochondrial genome of booklouse, Liposcelis sculptilis (Psocoptera: Liposcelididae) and the evolutionary timescale of Liposcelis. Sci. Rep. 6, 30660; doi: 10.1038/srep30660 (2016).

(c) (i) This work is licensed under a Creative Commons Attribution 4.0 International License. The images unless indicated otherwise in the credit line; if the material is not included under the Creative Commons license, users will need to obtain permission from the license holder to reproduce the material. To view a copy of this license, visit http://creativecommons.org/licenses/by/4.0/

(c) The Author(s) 2016 